\title{
Distinguishing cognitive effort and working memory load using scale-invariance and alpha suppression in EEG
}

Omid Kardan¹, Kirsten C. S. Adam²,3, Irida Mance ${ }^{4}$, Nathan W. Churchill5,6, Edward K. $\operatorname{Vogel}^{1,7,8}, \&$ Marc G. Berman ${ }^{1,8}$

1 Department of Psychology, University of Chicago, Chicago, IL

2 Department of Psychology, University of California San Diego, La Jolla, CA

${ }^{3}$ Institute for Neural Computation, University of California San Diego, La Jolla, CA

4 Department of Psychology, University of Oregon, Eugene, OR

${ }^{5}$ Neuroscience Research Program, St. Michael's Hospital, Toronto, ON, Canada

${ }^{6}$ Keenan Research Centre of the Li Ka Shing Knowledge Institute at St. Michael's Hospital, Toronto, ON, Canada

${ }^{7}$ Institute for Mind and Biology, University of Chicago, Chicago, IL

${ }^{8}$ Grossman Institute for Neuroscience, Quantitative Biology, and Human Behavior, University of Chicago, Chicago, IL

Short title: Scale-invariance and cognitive effort

Key words: working memory; cognitive effort; scale-invariance, EEG

Conflicts of Interest: None

Word count of intro + discussion: $1073+905=1978$ words

Funding: Research was supported by grants awarded to E.V. (National Institute of Mental Health grant 5R01-MH087214 and Office of Naval Research grant N00014-12-1-0972). K.A. was supported by National Institute of Mental Health grant 5T32-MH020002. M.G.B. was supported in part by a grant from the TKF Foundation, the National Science Foundation (BCS-1632445) and the John Templeton Foundation as well as internal grants from the University of Chicago.

\section{Correspondence:}

Omid Kardan: okardan@uchicago.edu or Marc Berman: bermanm@uchicago.edu

Department of Psychology,

University of Chicago,

5848 South University Avenue,

Chicago, IL, 60637 


\begin{abstract}
Despite being intuitive, cognitive effort has proven difficult to quantify. In the current study we validated the correspondence between scale-invariance $(H)$ of cortical activity recorded by EEG and task load during two working memory (WM) experiments with varying set sizes. We used this neural signature to disentangle cognitive effort from the number of items in WM. Our results showed monotonic decreases in $H$ with increased set size, even after set size exceeded WM capacity. This behavior of $H$ contrasted with behavioral performance and an oscillatory indicator of WM load (i.e., alpha-band desynchronization), both of which showed a plateau at difficulty levels surpassing WM capacity. This is the first reported evidence for the suppression of scale-invariance in EEG due to task difficulty, and our work suggest that $H$ suppression may be used to quantify changes in effort even when working memory load is constant and at maximum capacity.
\end{abstract}




\section{Introduction}

Cognitive effort is a seemingly intuitive aspect of cognition, yet has proven difficult to quantify because of gaps in both theory and practice. Some researchers, at least implicitly, equate mental effort with working memory (WM) load (Garbarino \& Edell, 1997; Kitzbichler, Henson, Smith, Nathan, \& Bullmore, 2011), whereas others see WM load and effort as confounded measures that could, in theory, be separated (Vogel \& Machizawa, 2004). Practically, the literature lacks a sensitive and specific neural signature that can be used as a marker of mental effort. As a result of these gaps, the literature has produced many competing operational definitions of effort (Otto \& Daw, 2018; Paas, Tuovinen, Tabbers, \& Gerven, 2003; Westbrook \& Braver, 2015). Therefore, the identification of a reliable neurobiological marker specific to cognitive effort would facilitate the operationalization of cognitive effort for both theoretical and applied research. Within the context of neuroimaging of WM tasks, a core challenge for identifying a neural signal of effort involves disentangling effort signals from WM storage signals. This is because, until maximum WM capacity is reached, exerting more effort often enables meeting larger WM demands. To address this challenge, we took advantage of results from two wellcharacterized research domains. By combining what is known from fMRI studies of effort signals with what is known from EEG studies of working memory storage signals, we were able to identify a specific neural marker of effort in EEG, distinct from working-memory storage signals.

fMRI research has revealed a potential neural signature for quantifying effort: scaleinvariance of the broadband signal. Scale-invariance in biological signals (sometimes also called scale-free or fractal scaling) refers to a property of signals where all measured time 
scales contribute to a signal of interest, with no particular timescale having a dominant contribution. It is a property of "long-memory" systems with persistent autocorrelations and is typically modeled as a power-law relationship between frequency $f$ and power spectral density $\operatorname{PSD}(f)$ of the signal, $\operatorname{PSD}(f) \sim|f|^{-\beta}$, for scaling parameter $\beta \geq 0$. This is often expressed in terms of the Hurst exponent $(H)$, which quantifies temporal dependence of signals, with higher values denoting a more scale-free signal. Scale-free dynamics are a feature of many biological systems under complex control including the human nervous system (Werner, 2010) and a breakdown of fractal scaling often signifies increased physiological stress (Goldberger et al., 2002); for other interpretations of $H$ in neural signals see von Wegner, Laufs, \& Tagliazucchi, 2018). Research on scale-free dynamics of fMRI timeseries demonstrates that global Blood Oxygenation Level Dependent (BOLD) activity of the brain becomes less scale-free (lower $H$ ) in the presence of cognitive tasks and other modulators of cognitive effort (Barnes, Bullmore, \& Suckling, 2009; Churchill et al., 2016; He, 2011). Specifically, fMRI timeseries have lower $H$ when: 1 ) performing hard vs. easy perceptual decision tasks, (Churchill et al., 2016), 2) performing a visual detection task vs. open-eyes rest (He, 2011), 3) performing n-back task versus rest (Barnes et al., 2009), 4) performing novel tasks vs. more familiar tasks (Churchill et al., 2016), and 5) when participants were under physical and mental burden (Churchill et al., 2015). These findings suggest that suppression of $H$ may be a generalizable neuroimaging marker of increased cognitive effort.

EEG research on visual working memory (VWM) points to a capacity limit (referred to as $K$ ) beyond which the individual cannot maintain any more mental representations of the presented items. Specifically, recent research in VWM suggests a limit of 3-4 items for $K$ 
(Adam, Vogel, \& Awh, 2017; Fukuda, Awh, \& Vogel, 2010; Luck \& Vogel, 2013; Rouder et al., 2008), but see (Alvarez \& Cavanagh, 2004)). Additionally, this research has suggested that the primary role of alpha band $(8-12 \mathrm{~Hz})$ oscillations $(\alpha)$ in the context of VWM is to maintain distinct working memory representations rather than to suppress irrelevant information (Foster \& Awh, 2019). This indicates that as the number of relevant items in VWM increases, $\alpha$ will systematically decrease until WM capacity $(K)$ is reached (Adam, Robison, \& Vogel, 2018; Fukuda, Kang, \& Woodman, 2016; Fukuda, Mance, \& Vogel, 2015). Therefore, translating the fMRI work examining $H$ and cognitive effort to EEG experiments on oscillatory activity and $K$ can provide us with the framework needed to investigate the distinction between cognitive effort and WM load. Pertinently, previous literature has shown that BOLD fMRI signal fluctuations are linked to scale-free fluctuations in EEG measurements of neural activity (Van de Ville, Britz, \& Michel, 2010). As such, an effortrelated suppression in scale-invariance would also be expected at the electrophysiological timescale. Additionally, studying these broadband effects with EEG will potentially be more fruitful than with fMRI since this method overcomes the limited range of timescales that can be examined for $H$ in fMRI due to its slow sampling rate. To our knowledge, this relationship between suppression of $H$ and cognitive effort beyond $K$ has not yet been investigated using EEG, where it would provide a critical validation of the fMRI findings using a more direct measure of neural activity and a wider range of accessible timescales.

In the current study, we analyzed two VWM EEG experiments including a 'Remember N' task (Experiment 1) and a Discrete Whole-Report task (Experiment 2)1․ In both experiments, a memory array was presented to participants while they maintained

\footnotetext{
${ }^{1}$ Aspects of experiment 1 and 2 were previously published in (Mance, 2015) and (Adam, Robison, \& Vogel,
} 2018), respectively. 
fixation on the center of the screen. Memory load was parametrically varied $(N=1,2,3$, or 6 items $)^{2}$. We hypothesized that scale-invariance $(H)$ of the EEG signal during the retention interval would decline parametrically with increasing task load, even beyond $K$ (i.e., beyond 3-4 items). In contrast, $\alpha$ suppression has been shown to plateau for set sizes beyond $K$. As such, we further predicted that the pattern of $H$ across set sizes would significantly diverge from the pattern of $\alpha$ across set sizes as $\alpha$ suppression would not continue to decrease beyond $K$. The above predictions assume cognitive effort to be distinguishable from the amount of information in WM. In summary, we propose that (1) global scale-invariance of cortical brain activity as recorded by EEG can be used as a metric of cognitive effort, and (2) this broad-band signal characteristic can dissociate VWM load from the effort exerted for the task at hand, thus providing a neurobiological dissociation between effort and information in WM.

\section{Experiment 1 Methods}

\section{Participants}

Experiment 1 included 31 participants (18-35 years old; 17 female) from the University of Oregon and surrounding community (Mance, 2015). A total of 6 were excluded from analyses because of excessive EEG artifacts (see Artifact Rejection), leaving a final sample of 25 participants. Participants gave written informed consent, and experimental procedures were approved by the University of Oregon's Committee for the Protection of Human Subjects (CPHS) and Institutional Review Board (IRB). All participants

\footnotetext{
${ }^{2}$ For experiment $2, \mathrm{~N}=1,3$, and 6 .
} 
had normal or corrected-to-normal visual acuity and reported no history of neurological disorders.

\section{Stimuli}

Participants were seated $\sim 100 \mathrm{~cm}$ from a 17 -in. cathode ray tube monitor. Stimuli were rendered using the Psychophysics toolbox (Brainard, 1997; Pelli, 1997). Participants remembered colored squares presented on a medium gray background (RGB = 120120 120) while maintaining fixation on a small white $\operatorname{dot}\left(0.2^{\circ}\right)$. A pool of eight distinct colors: red ( $R G B=2550$ 0), green ( 0255 0), blue ( 00255$)$, yellow (255 255 0), magenta (255 0 255), cyan (0 255 255), white (255 255 255), and black (1 11 1) were used to choose the colors from in each trial. Each square subtended $1^{\circ}$, and each square was placed at one of 32 locations on the screen (equally spaced grid of 4 x 8 locations, placed within a portion of the display subtending $8.1^{\circ}$ to the left or right of fixation and $4.7^{\circ}$ above or below fixation.

\section{The Remember N Task}

On each trial, participants were first given a cue which indicated how many items they should encode from the upcoming display (e.g., "Remember 3") and pressed the spacebar to initiate the trial. The cue presentation was un-speeded but had a minimum duration of $600 \mathrm{~ms}$. After a blank baseline (1,200 ms), participants briefly viewed six colored squares (150 ms) and remembered all or some of these squares across a delay (1,150 ms). Participants were instructed to try their best to only remember the cued number of items and to ignore the rest (Figure 1), but they were not instructed about which particular items to remember (they could freely choose). To respond, participants clicked the color in the response grid corresponding to the color remembered at that 
location. During the response, participants only had the option to respond to as many items as they were cued to remember (for example, if the cue was "Remember 1", they could only make one response during the recall phase). After the participant had made all responses the cue for the next trial appeared after $500 \mathrm{~ms}$. There were a total of 150 trials per set-size (30 blocks of 20 trials, $\sim 2.5$ hours).

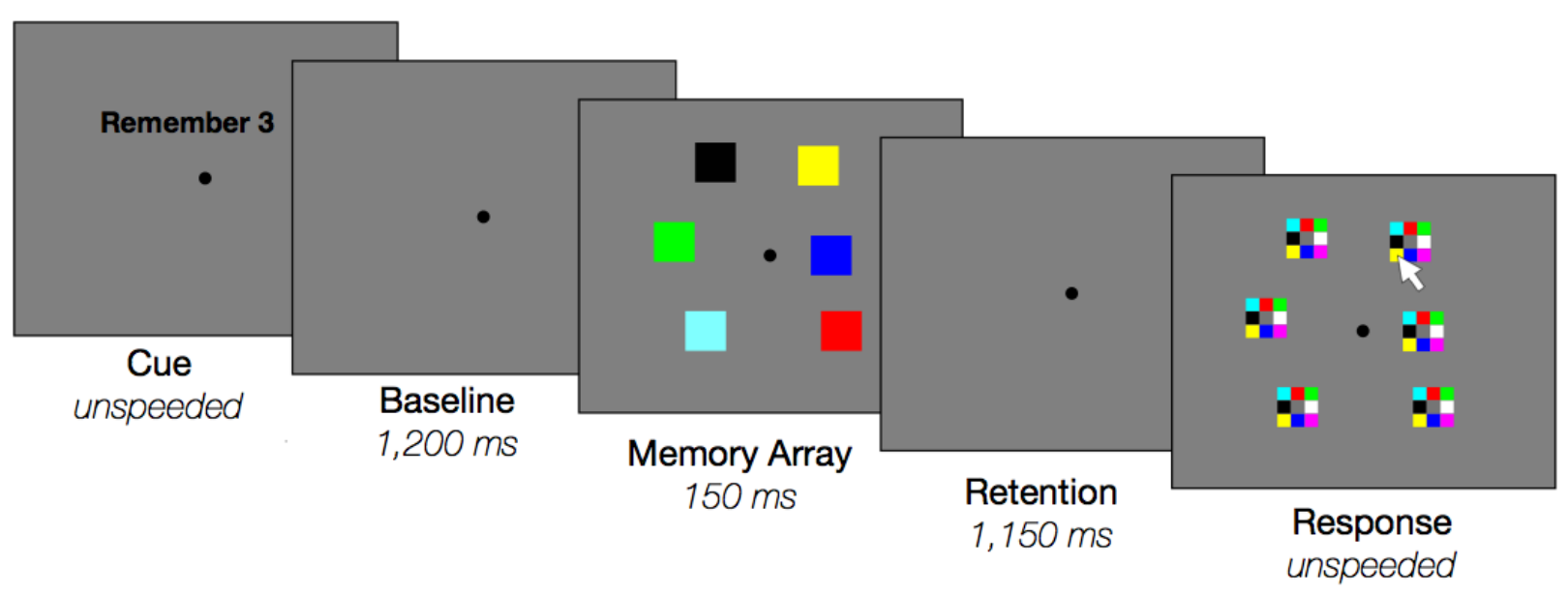

Figure1: Experiment 1: "Remember N" task procedures. At the beginning of every trial, participants were cued about how many items to remember from the upcoming memory array. Memory arrays always contained six items shown in random locations. At response, participants only recalled as many items as they were asked to remember.

\section{Data Acquisition and Analysis}

Data were acquired with an SA Instrumentation amplifier with a band-pass of .01-80 $\mathrm{Hz}$ and digitized at $250 \mathrm{~Hz}$ in LabView 6.1. We measured EEG from 20 tin electrodes mounted in an elastic cap (Electrocap International, Eaton, OH) at International 10/20 sites F3, Fz, F4, T3, C3, Cz, C4, T4, P3, Pz, P4, T5, 01, and 02 and five nonstandard sites: OL midway between T5 and 01, OR midway between T6 and 02, P03 midway between P3 and OL, P04 midway between P4 and OR, and POz midway between P03 and P04 (Figure S1). All sites were recorded using a left-mastoid reference and re-referenced offline to the algebraic average of the left and right mastoids. The vertical electrooculogram (EOG) was 
recorded from an electrode placed $\sim 1 \mathrm{~cm}$ below the right eye. The horizontal EOG was recorded from electrodes placed $\sim 1 \mathrm{~cm}$ lateral to the external canthus of each eye. Electrode impedances were kept below $3 \mathrm{k} \Omega$. Participants were instructed not to move their eyes or blink during the trial until the test array appeared on the screen. Trials including horizontal eye movements, blinks, blocking (amplifier saturation after drift), or excessive noise were excluded from analysis (mean $=16.8 \%$ of trials).

\section{Artifact Rejection}

For horizontal eye movement rejection, we used a split-half sliding window approach (window size $=150 \mathrm{~ms}$, step size $=10 \mathrm{~ms}$, threshold $=20 \mu \mathrm{V}$ ) on the HEOG signal, where a $150 \mathrm{~ms}$ time window was slid in steps of $10 \mathrm{~ms}$ from the beginning to the end of the trial. If the change in voltage from the first half to the second half of the window was greater than $20 \mu \mathrm{V}$, it was marked as an eye movement and rejected. We also used a sliding window step function to check for blinks in the VEOG (window size $=100 \mathrm{~ms}$, step size $=10$ ms, threshold $=40 \mu \mathrm{V}$ ). For blocking rejection, we slid a $200 \mathrm{~ms}$ time window in steps of 50 ms and excluded trials for blocking if any EEG electrode had at least 20 consecutive timepoints (i.e. $80 \mathrm{~ms}$ ) that were within $1 \mu \mathrm{V}$ of each other. We excluded trials for excessive noise if any electrode had peak-to-peak amplitude greater than $200 \mu \mathrm{V}$ within a $15 \mathrm{~ms}$ time window. Data were visually inspected to confirm that rejection criteria were working as expected; some additional trials were manually rejected. Six participants were excluded for excessive artifacts (fewer than 70 good trials per condition on average, remaining $n=25$ ). After rejection, included participants had an average of 110 trials per condition (SD = 22.5). 


\section{Estimation of $\alpha$ Power}

To estimate alpha power (8-12 Hz), we first band-pass filtered the raw EEG signal for each trial and electrode using a two-way least-squares FIR filter , 'eegfilt.m', (Delorme \& Makeig, 2004). We calculated instantaneous power with the MATLAB Hilbert transform ('hilbert.m'). Because we were interested in event-related changes to alpha power as a function of memory load, we analyzed percent change in alpha power relative to the memory array onset. For each participant and electrode, we first averaged single-trial power across each load condition, then calculated the percent change in alpha power for each condition relative to a baseline period (500 ms to $100 \mathrm{~ms}$ before the memory array).

\section{Estimation of Scale-Invariance $(H)$}

Scaling analyses of temporal signals seek to determine whether all measured frequencies (or timescales) contribute to the signal of interest. Scale-invariance occurs when no particular frequency (or timescale) has a dominant contribution to the signal, implying that the signals have long-range temporal dependency. This dependency is indexed via the Hurst exponent $H$, where $H \leq 0.5$ indicates short-range dependency, $H=0.5$ denotes an uncorrelated process, and $H \geq 0.5$ indicates long-range dependency, with $H=1$ indicating complete scale-invariance.

While there exist many different methods to estimate $H$, the wavelet leader multifractal (WLMF) formalism has emerged as a powerful technique that is highly efficient and robust to signal non-stationarity (Jaffard, Lashermes, \& Abry, 2007). We applied the WLMF method on the EEG signal during retention interval (after memory array and before response) to quantify the scale-invariance of the EEG signal during maintenance. The 
wavelet transform uses translated and dilated versions of a basis function $\Psi([t-k] / a)$ to analyze the signal of interest at different delays and time scales. The wavelet coefficient $d_{x}(a, k)$ measures signal energy present at delay $k$ and time scale $a$ by calculating the integral:

$$
d_{x}(a, k)=\frac{1}{a} \int x(t) \Psi\left(\frac{t-k}{a}\right) d t
$$

At a range of dyadic scales, i.e., $a=2^{j}$ for integer $j$. Wavelet leaders $L_{x}(a, k)$ are subsequently calculated as the largest coefficient value $\left|d_{x}\left(a^{\prime}, k^{\prime}\right)\right|$ within a narrow temporal neighbourhood of $k$, for any scale $a^{\prime} \leq a$. Multifractal scaling is then defined by the function:

$$
\frac{1}{K} \sum_{k}\left|L_{x}\left(2^{j}, k\right)\right|^{q}=C_{q} 2^{j \varsigma(q)}
$$

Which describes wavelet power as a function of time scale, for a range of different scaling exponents $q$, in terms of a characteristic function $\varsigma(q)$. Typically parameterized as a polynomial expansion $\varsigma(q)=\sum_{p} c_{p}\left(q^{p} / p !\right)$, the log-cumulants $c_{\mathrm{p}}$ define the scaling behavior of the signal $x(t)$. In this study, we focused on first-order cumulant $c_{1}$, which is closely linked to the monofractal scaling parameter $H$ (Wendt, Abry, \& Jaffard, 2007). An exploratory analysis of the higher-order cumulants ( $c_{2}$ and $\left.c_{3}\right)$ did not show a relationship between them and the memory load in our two experiments.

\section{Canonical Correlation Analysis}

In a canonical correlation analysis (CCA), two sets of variables are related together (as opposed to many variables to a single dependent variable) and the degree of relationship between the two sets of variables is assessed (Hotelling, 1936). An 
advantage of multivariate methods such as CCA to univariate methods is that they reveal the linear relationship between all spatial (every electrode) and task-related (every task load level) contrasts simultaneously without the multiple comparison problem or the need for coming up with a-priori contrasts.

For two datasets $\mathrm{X}$ and $\mathrm{Y}, \mathrm{CCA}$ identifies pairs of canonical weighting vectors $\mathrm{w}_{\mathrm{X}}$ and $\mathrm{w}_{\mathrm{y}}$ which produce corresponding linear latent variables $I_{\mathrm{x}}=\mathrm{Xw}_{\mathrm{x}}$ and $l_{\mathrm{y}}=\mathrm{Yw}_{\mathrm{y}}$, such that correlation $\rho_{\mathrm{XY}}=\operatorname{corr}\left(l_{\mathrm{x}}, l_{\mathrm{y}}\right)$ is maximized and orthogonal to any other latent variable pairs. We used CCA to find latent variables that relate either scale-invariance $(H)$ of the 20 electrodes (bottom set of variables in Figure 2A) to the task loads (top set of variables in Figure 2A) or the alpha desynchronization of the 20 EEG electrodes (bottom set of variables in Figure 2B) to the task loads (top set of variables in Figure 2B). Each task load (i.e., set size) was dummy coded as a binary variable, so the number of total latent variables possible was $\mathrm{N}-1$ (i.e., the rank of the smaller set of variables), where $\mathrm{N}$ is the number of levels of load in each experiment ( $N=4$ in experiment $1 ; N=3$ in experiment 2). Only the primary latent variables in the experiments are shown in the results because no secondary (or tertiary for Exp. 1) latent variables were large enough $\left(\rho_{\mathrm{XY}}>0.25\right)$ in either experiment to be considered meaningful. Small effects, conventionally in the range of $\mathrm{r}=$ [0.15 0.25] (Kirk, 2012), are not of interest here because a biomarker with smaller than medium effect size (i. e., $r=0.25$ ) (Kirk, 2012) will likely not have practical utility due to lack of sufficient sensitivity. Each electrode's or task load's contribution to the latent variable is represented by the canonical weight of the observed variable which is the Pearson correlation coefficient of that variable with the latent variable. The stability of the latent variables is evaluated by the error bars of the canonical weights on both left 
and right sets, which are the $95 \%$ confidence intervals calculated by bootstrapping the data (3000 samples with replacement) to create distributions for the canonical weights similar to (Kardan et al., 2017). The strength of the association between the two sets in a latent variable is represented by the correlation between the two sides (double sided arrows in Figure 2). As such, the relationship represented in a latent variable is considered strong and stable if the correlation is large $\left(\rho_{\mathrm{XY}}>0.4\right)$ and there is at least one variable in each side (i.e., at least one electrode and one task load) whose weight reliably differs from zero across the bootstrapped samples (i. e., omnibus non-parametric $\mathrm{p}<0.05$ ).

\section{Statistical Power}

Following our hypothesis regarding relationships between electrode data and set sizes, we simulated the CCA analysis to estimate statistical power for detecting a true correlation between electrodes' data and a contrast delineating a set size from the other set sizes (e.g., contrast $C=[-1-1+1-1]$ delineates set size 3 in Experiment 1 ). This was done in 5 steps. First, we generated a random Gaussian variable Norm $(0,1)$ for $N$ levels of set size and 20 electrodes for $n$ participants. Second, to create a true correlation between the generated electrodes data and a set size contrast, we introduced a bias (i. e., a uniformly distributed random variable with an expected value above zero) in the generated electrode data for one set size (chosen randomly). We did this by adding a $\beta_{\text {bias }} *$ Unif $(0,1)$ to the generated data for that set size and subtracting $\beta_{\text {bias }} *$ Unif $(0,1)$ from the other set sizes. This produces a difference of $\beta_{\text {bias }}$ in the expected values of the electrode data for the biased set size compared to electrode data of other set sizes. Third, through trial and error iterations, we found that $\beta_{\text {bias }}=0.62$ induced correlations that closely resembled the minimum effect size of interest ( $r=0.25$, see CCA analysis). This value of $\beta_{\text {bias }}$ resulted in 
true correlations in the range of $r=\left[\begin{array}{ll}0.24 & 0.26\end{array}\right]$ between the set size contrast (i. e., dummy variables coding 1 for the biased set size and -1 for the other set sizes) and the generated electrodes data for Experiment 1. Fourth, we then applied the CCA procedure explained in the previous section ${ }^{3}$ to the generated data to determine whether the CCA weight for the biased set size in the primary latent variable was found to be significant at $\alpha=0.05$. Finally, we repeated step (4) 500 times, with $N=4$ and $n=25$ to simulate the final sample size for Experiment 1 . Statistical power was estimated to be 0.78 by calculating the proportion of times in the 500 runs where the induced correlation was found to be significant in the simulated CCAs.

\section{Experiment 1 Results}

The average number of correctly reported items was $0.96(\mathrm{SD}=0.03)$ items, 1.84 $(\mathrm{SD}=0.13)$ items, $2.35(\mathrm{SD}=0.35)$ items, and $2.29(\mathrm{SD}=0.44)$ items for set sizes $1,2,3$, and 6, respectively. This pattern of performance is consistent with our assumption that working memory capacity in this task would be limited to around 3 items; when the set size increased from 3 to 6 items, the number of correctly reported items did not increase (decrease of 0.06 items, $\mathrm{p}=0.771$ ).

\section{CCA Results for Scale-Invariance}

In the Remember $\mathrm{N}$ task, the primary latent variable from the CCA showed a strong $\left(\mathrm{R}^{2}=0.254, \mathrm{p}<0.001\right.$ ) and stable relationship (see Figure $2 \mathrm{~A}$ bottom panel) between the number of to-be-remembered items and the scale-invariance of the EEG signal during retention, where a global decrease in $H$ (especially in occipital and parietal regions, see

\footnotetext{
${ }^{3}$ The only difference being that instead of 3000 resamples for each CCA, we did 300 resamples to cut down processing time in each loop of the simulation.
} 
Figure 2B) tracked increases in task load. Importantly, the decrease in scale-invariance corresponded monotonically to task load, where each load level had a significantly larger canonical weight than the previous one (except for when going from load 1 to 2, perhaps due to both loads being relatively easy). The canonical weights were $r_{1}=-0.63, r_{2}=-0.39, r_{3}$ $=0.24$, and $r_{6}=0.78$ for each set size, respectively. The significant increment from load 3 to 6 (one-sided arrow in Figure 2A top) follows our hypothesis that $H$ is sensitive to the exerted cognitive effort rather than the capacity limits of visual working memory.

\section{CCA Results for $\alpha$ Desynchronization}

As shown in Figure 2C, the primary CCA latent variable relating change in $\alpha$ power during the retention period and task load showed a strong and stable relationship $\left(\mathrm{R}^{2}=\right.$ $0.239, \mathrm{p}<0.001$ ) between the two sets of variables. As expected, lower $\alpha$ power in the posterior electrodes (occipital and parietal, see Figure 2D) corresponded to higher task load. The canonical weights were $r_{1}=-0.83, r_{2}=-0.09, r_{3}=0.66$, and $r_{6}=0.25$ for each set size, respectively. Importantly, the correspondence was not monotonic as indicated by the canonical weight for load 6 not being larger than canonical weight for load 3 (in fact it was marginally smaller; non-parametric $\mathrm{p}=0.057$ ). Thus, the relationship between alpha power and task load diverged from the monotonic correspondence between $H$ and task load that we reported above. This bifurcation (see one-sided arrows in Figure 2A and C top panels) in the relationship between task load and $H$ versus alpha was statistically significant (Fischer's $\mathrm{Z}=2.66, \mathrm{p}=0.008$ ), supporting our hypothesis that posterior $\alpha$ desynchronization is sensitive to working memory load rather than cognitive effort. Together, the results from the first experiment suggest that working memory load and 
cognitive effort are distinguishable and can be tracked by alpha band desynchronization versus broad-band characteristics of the EEG signal $(H)$.
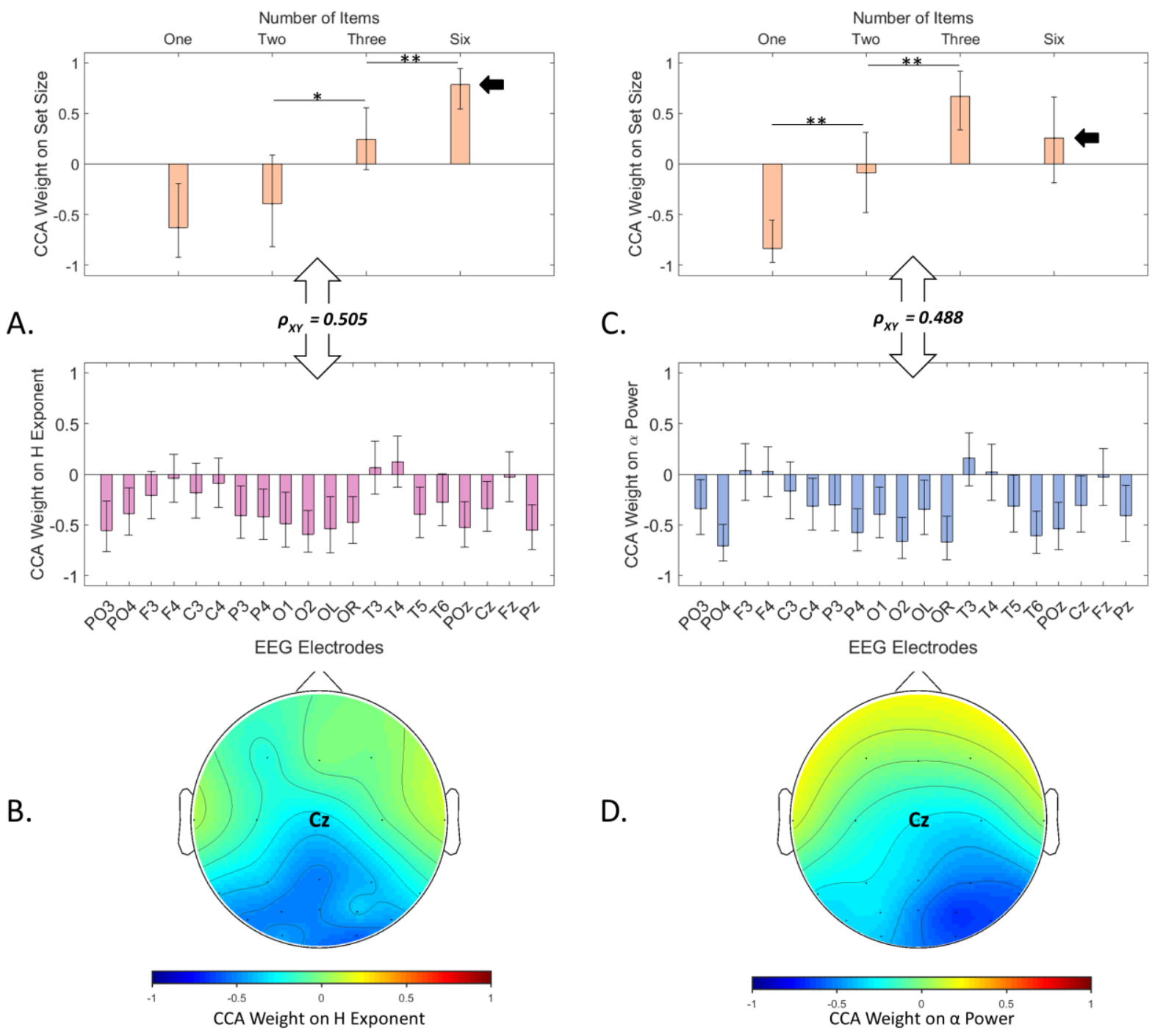

Figure 2. The primary latent variables of the CCA analysis for the Remember $\mathrm{N}$ task in Experiment 1. (A) The primary relationship between scale-invariance $(H)$ of the EEG electrodes during the retention period (pink) with the number of items to remember (orange). The double-headed arrows show the strength of the correlation between the bottom set (electrodes) and the top set (task loads). The vertical axis in each panel shows the contribution of an electrode (bottom) or a specific task load (top) to the latent variable as indicated by the size of its normalized CCA weight. The error bars show bootstrapped 95\% confidence intervals around the means for the weights. The asterisks show statistical significance in the difference between weights of adjacent levels of task load (i.e., 1 item to 
2 items, 2 items to 3 items, and 3 items to 6 items), ${ }^{*} p<0.05$ and ${ }^{* *} p<0.01$. (B)

Topographic demonstration of the CCA weights on the $H$ exponent. (C) The primary relationship between the desynchronization of $\alpha$ band power from baseline during the retention period (blue) with the number of items to remember (orange). One-sided arrows demonstrate difference in CCA weight for set size 6 in the relationship between task load and $H$ versus task load and alpha power. (D) Topographic demonstration of the CCA weights on alpha power.

\section{Experiment 2 Methods}

\section{Participants}

A group of 31 participants (ages between 18 to 35, 12 women) were recruited from the University of Oregon and surrounding community (Adam et al., 2018). All participants had self-reported normal or corrected-to-normal visual acuity and normal color vision. All participants gave informed consent and completed the 3-hr session for $\$ 30$ in compensation. Four participants were excluded from analyses for having fewer than 70 trials per condition on average after artifact rejection (remaining $n=27$ ). After rejection, included participants had an average of 148 trials per condition (SD = 39.5).

\section{Stimuli}

Stimuli and procedures were similar to Experiment 1. The key difference was that that for this lateralized whole-report task, participants were cued to attend either the leftor right-half of the display before the onset of the memory array. They were asked to remember all items presented in the cued hemifield. The cue was a small pink and green diamond, approximately $0.2^{\circ}$ tall by $0.4^{\circ}$ wide and was presented $0.4^{\circ}$ above the fixation cross. Participants remembered colored squares presented on a medium gray background $(\mathrm{RGB}=127.5127 .5127 .5)$ while maintaining fixation on a small black dot $\left(0.12^{\circ}\right)$. A pool of nine distinct colors: red (RGB = 2550 0), green (0 255 0), blue ( 00 255), yellow (255 255 
0), magenta (255 0 255), cyan (0 255 255), orange (255 128 0), white (255 255 255), and black ( $\left.\begin{array}{lll}1 & 1 & 1\end{array}\right)$ were used to choose the colors from in each trial. Each square subtended $1.2^{\circ}$ and they could appear anywhere within a portion of the display subtending $7.0^{\circ}$ to the left or right of fixation and $5.2^{\circ}$ above or below fixation as long as there was a minimum distance of at least 1.5 squares between the centroids of any two squares.

\section{Discrete Whole-Report Task}

Each trial began with a blank inter-trial interview (500 ms) followed by the spatial cue (1100 ms) which indicated which side of the screen to attend. After the cue period ended, a memory array containing an equal number of items on both sides of the screen (cued and uncued) was presented for 250 ms. The colors of squares in the memory array were chosen without replacement within each side (i.e., all cued colors were unique but might be repeated on the uncued side of the display). After encoding, participants remembered the items across a blank delay of $1300 \mathrm{~ms}$. At test, a $3 \times 3$ matrix of the nine possible colors was presented at the location of each item on both the attended and unattended side. Similar to the Remember N task in Experiment 1, participants were instructed to click the color in each matrix corresponding to the color presented at the location (see Figure 3). The response period ended after participants made a response for all items on the attended side. Participants clicked the mouse to initiate the beginning of the next trial. 


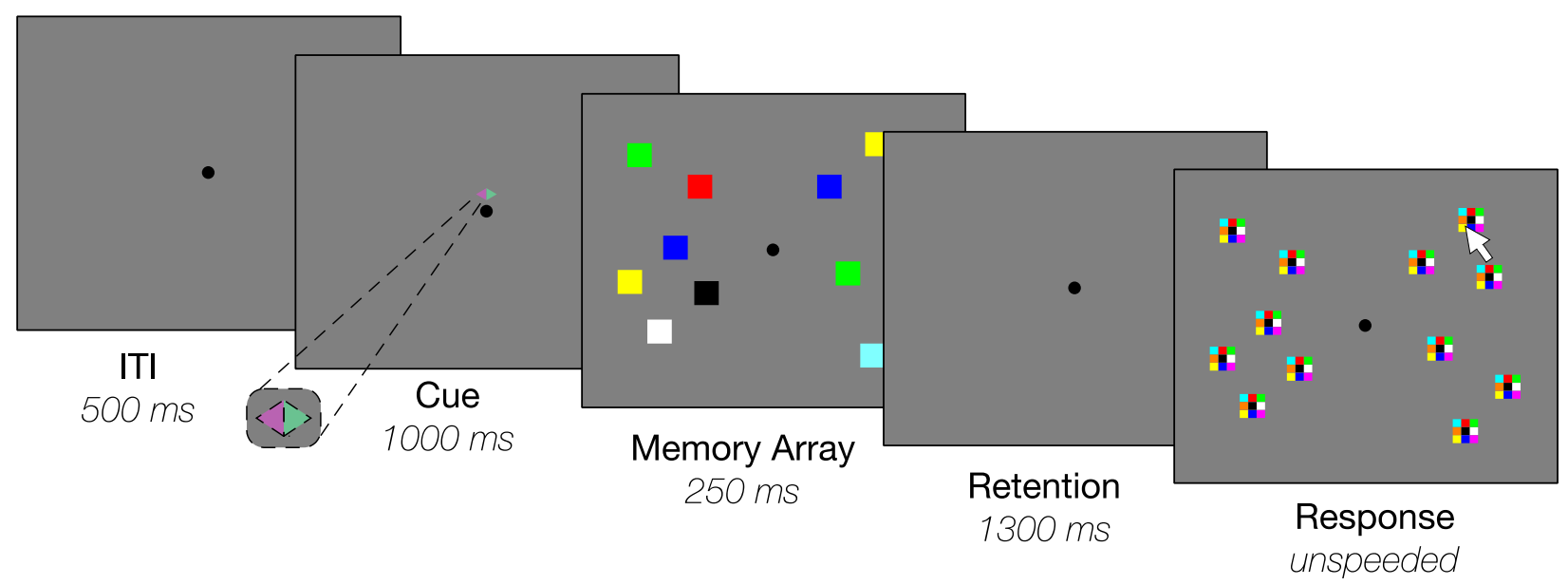

Figure 3. Experiment 2 "Whole-Report" task procedures. At the beginning of every trial participants were given a cue indicating which side of the screen to remember from the upcoming memory array. Memory arrays could contain 1, 3, or 6 items shown in random locations on each side; at response participants only recalled items from the cued side.

\section{Data Analysis and Statistical Methods}

Artifact rejection, alpha power analysis, wavelet analysis for $H$, and the CCA analysis were the same as described in Experiment $1^{4}$. For statistical power, the parameters of the simulation were set at $\mathrm{N}=3, \mathrm{n}=27$, and $\beta_{\text {bias }}=0.60$ for Experiment 2 , which determined a statistical power of 0.72 for the analysis. The simulation showed that Experiment 2 would have sufficient power (conventionally 0.8 ) to detect effects only if they are equal to or larger than $r=0.29$.

\section{Experiment 2 Results}

The average number of correctly reported items was $0.95(S D=0.04)$ for set size 1 , $2.41(\mathrm{SD}=0.33)$ for set size 3 , and $2.53(\mathrm{SD}=0.53)$ for set size 6 . This, again, indicates that average $K$ was still less than 3 even for 6 items, although performance was slightly better for 6 items (0.12 items increase, $\mathrm{p}=0.085)$.

\footnotetext{
${ }^{4}$ Analysis where we only calculated alpha desynchronization for trials ipsilateral to lateralized electrodes yielded very similar results.
} 


\section{CCA Results for Scale-invariance}

Replicating the Remember $\mathrm{N}$ task, the primary latent variable from the CCA in the lateralized Whole-Report task showed a very strong $\left(\mathrm{R}^{2}=0.416, \mathrm{p}<0.001\right)$ and stable relationship between the number of presented items to be remembered in the task and the scale-invariance of the EEG signal during retention (Figure 4A). Again, a global decrease in $H$ tracked increases in the task load. The canonical weights for each set size in this latent variable were $r_{1}=-0.78, r_{3}=-0.13$, and $r_{6}=0.91$, respectively. Importantly, the decrease in scale-invariance changed monotonically with task load, where the canonical weight for each load level was significantly larger than the previous one including from load 3 to 6. This follows our hypothesis that $H$ is sensitive to the exerted cognitive effort in visual working memory tasks.

\section{CCA Results for $\alpha$ Desynchronization}

The primary CCA latent variable relating change in $\alpha$ power and task load was not stable, as the $95 \%$ confidence intervals for all of the electrodes contained zero (Figure 4C bottom). The task load levels were not significantly different from one another (Figure 4C top), although there was a numerical trend of suppression of $\alpha$ power for 6 items compared to 1 item. Thus, the relationship between task load and alpha power did not follow the pattern we observed in Experiment 1. However, since the latent variable was not stable, the canonical correspondence for $\alpha$ suppression in this experiment was not conclusive with regards to our hypothesis about distinguishing working memory load from cognitive effort. 

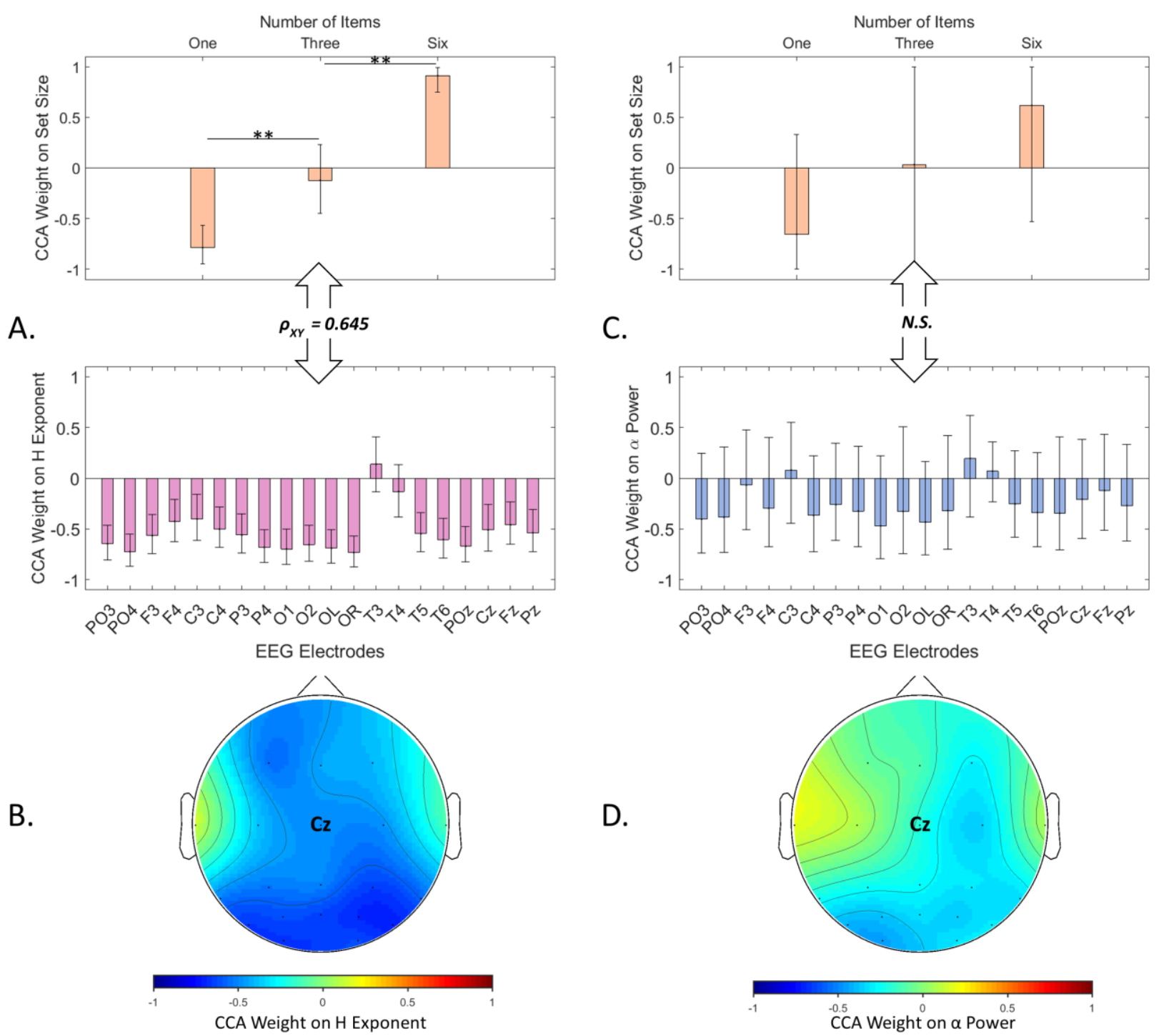

Figure 4. The primary latent variables of the CCA analysis for the Whole-Report task in Experiment 2. (A) The primary relationship between the scale-invariance $(H)$ of the EEG electrodes during retention period (pink) with the number of items to remember (orange). The double-headed arrows show the strength of the correlation between the bottom set (electrodes) and the top set (task loads). The vertical axis in each panel shows the contribution of an electrode (bottom) or a specific task load (top) to the latent variable as indicated by the size of its normalized CCA weight. The error bars show bootstrapped $95 \%$ confidence intervals around the means for the weights. The asterisks indicate statistical significance in the difference between weights of adjacent levels of task load (i.e., 1 item to 2 items, 2 items to 3 items, and 3 items to 6 items), where ${ }^{*} p<0.05$ and ${ }^{* *} p<0.01$. (B) Topographic demonstration of the CCA weights on the $H$ exponent. (C) The primary relationship between the desynchronization of $\alpha$ band power from baseline during retention period (blue) with the number of items to remember (orange). (D) Topographic demonstration of the CCA weights on alpha power. 


\section{Discussion}

In this study, we found a monotonic correspondence between scale-invariance of cortical activity recorded by EEG and task load across two experiments. This is the first reported evidence for the suppression of scale-invariance due to task difficulty as measured with EEG (a relatively high frequency signal), lending support to the previous literature relating fractalness of BOLD activity (a very low frequency signal) to task difficulty. Importantly, we used this signal characteristic in an attempt to address an elusive question of what distinguishes an effortful task from an easy one, and whether, in the context of doing a visual working memory task, there is more to cognitive effort than working memory load (Heitz, Schrock, Payne, \& Engle, 2008). Our results suggest that increases in $H$ with task load may be contrasted with behavioral performance, where the number of recalled items hardly increased after intermediate difficulty levels, if not completely plateaued. This suggests that $H$ is more sensitive to neural processes associated with task difficulty than with overt performance. Consequently, by showing a neurobiological dissociation between cognitive effort and the amount of information available for manipulation in working memory, our results support the theoretical account of cognitive effort that distinguishes it from information in working memory. One potential explanation for why an effortful state would be reflected in $H$ suppression of brain signals is the loss of self-organized criticality exhibited by a healthy brain in neutral state (Kitzbichler, Smith, Christensen, \& Bullmore, 2009) due to disproportionate allocation of resources to a specific cognitive demand. When $H$ is not suppressed, small perturbations can lead to large changes in brain state (R. Chialvo, 2004), thereby enabling rapid adaptability to heterogeneous external demands (Gisiger, 2001). Locking into an effortful 
state sacrifices the ability of the brain to adapt to other demands represented by a loss of criticality in brain networks, which exhibits itself in $H$ suppression.

Unlike $\alpha$ suppression, the differences in the design of the two experiments did not change the behavior of $H$ with regards to task load. Specifically, the differences in the experimental designs could test if certain aspects of the tasks such as the perception of items, the number of internal representations, or the inhibition of the distracting information is responsible for the suppression of scale-invariance. For example, in the Remember $\mathrm{N}$ experiment when participants were cued to only remember one item, the other five items on the screen were essentially distractors. If scale-invariance was primarily driven by inhibition of irrelevant information, the relationship between set size and $H$ should have been in the opposite direction between the two experiments, since the greatest amount of suppression is required in set-size 1 trials in Experiment 1 (five distractors) and set-size 6 in Experiment 2 (six distractors). If $H$ was modulated by the visual display imbalance between the different set sizes, then we should have only seen a correspondence between scale-invariance and set size in the whole-report task (Experiment 2) and not in Experiment 1 since the number of items on the screen was constant (6 items).

We found $H$ suppression to be more related to task difficulty than to task performance. Intuitively, factors such as motivation, fatigue, and anxiety can play roles in increased cognitive exertion in the absence of any specific improvements in behavior. The logic here is that higher levels of motivation, and/or lower levels of fatigue or anxiety, can increase the maximum capacity for expending effort differentially from their effect on working memory capacity (if any, see Bonner \& Sprinkle, 2002; Botvinick \& Braver, 2015). 
Previous fMRI studies have shown that fatigue due to physical burdens such as chemotherapy could disrupt brain function in a manner that is unrelated to working memory performance (Kardan et al., 2019). Moreover, such physical burdens in this cohort have been shown to alter the scaling properties of fMRI timeseries (Churchill et al., 2015).

Importantly, the scale-invariance of EEG signals in our experiments is a broad-band characteristic of the signal and even though it is (anti)correlated with narrow-band oscillations such as theta, alpha, and beta band power of the EEG signal (see supplementary Figure S2), its relationship with task difficulty was not driven by any of these specific narrow-band oscillation correlates (regarding cognitive tasks modulating beta and theta oscillations see (Engel \& Fries, 2010; Sederberg, Kahana, Howard, Donner, \& Madsen, 2003). We demonstrated this by applying CCA to investigate the correspondence between theta $(4-7 \mathrm{~Hz})$ as well as beta $(13-30 \mathrm{~Hz})$ band powers of the electrodes with the set sizes in both experiments similar to the analyses done for $\alpha$ and $H$. Neither theta nor beta band powers mimicked $H$ suppression's relationship with set size (see supplementary results: CCA for theta and beta bands).

In conclusion, in this study we used the time scale-invariance characteristic of the EEG signal $(H)$ to dissociate visual working memory load from the cognitive effort exerted for the task at hand. Our work further demonstrated the utility of $H$ as a neuromarker of cognitive effort. These results have theoretical implications for research on motivation and cognitive fatigue, as well as practical implications for education. Regarding theory, we ruled out interpretations of cognitive effort that equate it with working memory load. Regarding application, the relatively accessible $H$ of EEG signal can serve as robust neuro-feedback in educational interventions by indicating individual differences in effort towards solving 
specific problems, as well as tracking cognitive fatigue over time and thus signaling changes in motivation within an individual.

Author Contributions: M.G.B., K.A. and O.K. developed the hypothesis. N.C. wrote and developed custom Matlab scripts for analyzing scale-invariance. O.K. and K.A. analyzed the data. O.K. drafted the manuscript, and all authors edited and revised manuscript drafts.

Acknowledgements: We thank Matthew K. Robison, Richard Matullo, Will McGuirk, and Zhilong (Joshua) Wu for assistance collecting the published data that were used in Experiment 2 .

Data availability: All data will be made available on the Open Science Framework upon publication.

\section{References}

Adam, K. C. S., Robison, M. K., \& Vogel, E. K. (2018). Contralateral Delay Activity Tracks Fluctuations in Working Memory Performance. Journal of Cognitive Neuroscience, 30(9), 1229-1240. https://doi.org/10.1162/jocn_a_01233

Adam, K. C. S., Vogel, E. K., \& Awh, E. (2017). Clear evidence for item limits in visual working memory. Cognitive Psychology, 97, 79-97. https://doi.org/10.1016/j.cogpsych.2017.07.001

Alvarez, G. A., \& Cavanagh, P. (2004). The Capacity of Visual Short-Term Memory is Set Both by Visual Information Load and by Number of Objects. Psychological Science, 15(2), 106-111. https://doi.org/10.1111/j.0963-7214.2004.01502006.x 
Barnes, A., Bullmore, E. T., \& Suckling, J. (2009). Endogenous Human Brain Dynamics Recover Slowly Following Cognitive Effort. PLOS ONE, 4(8), e6626. https://doi.org/10.1371/journal.pone.0006626

Bonner, S. E., \& Sprinkle, G. B. (2002). The effects of monetary incentives on effort and task performance: theories, evidence, and a framework for research. Accounting, Organizations and Society, 27(4), 303-345. https://doi.org/10.1016/S03613682(01)00052-6

Botvinick, M., \& Braver, T. (2015). Motivation and Cognitive Control: From Behavior to Neural Mechanism. Annual Review of Psychology, 66(1), 83-113. https://doi.org/10.1146/annurev-psych-010814-015044

Brainard, D. H. (1997). The Psychophysics Toolbox. Spatial Vision, 10(4), 433-436. https://doi.org/10.1163/156856897X00357

Churchill, N. W., Cimprich, B., Askren, M. K., Reuter-Lorenz, P. A., Jung, M. S., Peltier, S., \& Berman, M. G. (2015). Scale-free brain dynamics under physical and psychological distress: Pre-treatment effects in women diagnosed with breast cancer. Human Brain Mapping, 36(3), 1077-1092. https://doi.org/10.1002/hbm.22687

Churchill, N. W., Spring, R., Grady, C., Cimprich, B., Askren, M. K., Reuter-Lorenz, P. A., ... Berman, M. G. (2016). The suppression of scale-free fMRI brain dynamics across three different sources of effort: aging, task novelty and task difficulty. Scientific Reports, 6, 30895. https://doi.org/10.1038/srep30895

Delorme, A., \& Makeig, S. (2004). EEGLAB: an open source toolbox for analysis of singletrial EEG dynamics including independent component analysis. Journal of 
Neuroscience Methods, 134(1), 9-21.

https://doi.org/10.1016/j.jneumeth.2003.10.009

Engel, A. K., \& Fries, P. (2010). Beta-band oscillations-signalling the status quo? Current Opinion in Neurobiology, 20(2), 156-165.

https://doi.org/10.1016/j.conb.2010.02.015

Foster, J. J., \& Awh, E. (2019). The role of alpha oscillations in spatial attention: limited evidence for a suppression account. Current Opinion in Psychology, 29, 34-40. https://doi.org/10.1016/j.copsyc.2018.11.001

Fukuda, K., Awh, E., \& Vogel, E. K. (2010). Discrete capacity limits in visual working memory. Current Opinion in Neurobiology, 20(2), 177-182. https://doi.org/10.1016/j.conb.2010.03.005

Fukuda, K., Kang, M.-S., \& Woodman, G. F. (2016). Distinct neural mechanisms for spatially lateralized and spatially global visual working memory representations. Journal of Neurophysiology, 116(4), 1715-1727. https://doi.org/10.1152/jn.00991.2015

Fukuda, K., Mance, I., \& Vogel, E. K. (2015). $\alpha$ Power Modulation and Event-Related Slow Wave Provide Dissociable Correlates of Visual Working Memory. Journal of Neuroscience, 35(41), 14009-14016. https://doi.org/10.1523/JNEUROSCI.500314.2015

Garbarino, E. C., \& Edell, J. A. (1997). Cognitive Effort, Affect, and Choice. Journal of Consumer Research, 24(2), 147-158. https://doi.org/10.1086/209500

Gisiger, T. (2001). Scale invariance in biology: coincidence or footprint of a universal mechanism? Biological Reviews, 76(2), 161-209. https://doi.org/10.1017/S1464793101005607 
Goldberger, A. L., Amaral, L. A. N., Hausdorff, J. M., Ivanov, P. C., Peng, C.-K., \& Stanley, H. E. (2002). Fractal dynamics in physiology: Alterations with disease and aging. Proceedings of the National Academy of Sciences, 99(suppl 1), 2466-2472. https://doi.org/10.1073/pnas.012579499

He, B. J. (2011). Scale-Free Properties of the Functional Magnetic Resonance Imaging Signal during Rest and Task. Journal of Neuroscience, 31(39), 13786-13795. https://doi.org/10.1523/JNEUROSCI.2111-11.2011

Heitz, R. P., Schrock, J. C., Payne, T. W., \& Engle, R. W. (2008). Effects of incentive on working memory capacity: behavioral and pupillometric data. Psychophysiology, 45(1), 119129.

Hotelling, H. (1936). Relations Between Two Sets of Variates. Biometrika, 28(3/4), 321-377. https://doi.org/10.2307/2333955

Jaffard, S., Lashermes, B., \& Abry, P. (2007). Wavelet Leaders in Multifractal Analysis. In T. Qian, M. I. Vai, \& Y. Xu (Eds.), Wavelet Analysis and Applications (pp. 201-246). https://doi.org/10.1007/978-3-7643-7778-6_17

Kardan, O., Reuter-Lorenz, P. A., Peltier, S., Churchill, N. W., Misic, B., Askren, M. K., ... Berman, M. G. (2019). Brain connectivity tracks effects of chemotherapy separately from behavioral measures. NeuroImage: Clinical, 101654. https://doi.org/10.1016/j.nicl.2019.101654

Kardan, O., Shneidman, L., Krogh-Jespersen, S., Gaskins, S., Berman, M. G., \& Woodward, A. (2017). Cultural and Developmental Influences on Overt Visual Attention to Videos. Scientific Reports, 7(1), 11264. https://doi.org/10.1038/s41598-017-11570-w 
Kirk, R. E. (2012). Experimental Design. In Handbook of Psychology, Second Edition. https://doi.org/10.1002/9781118133880.hop202001

Kitzbichler, M. G., Henson, R. N. A., Smith, M. L., Nathan, P. J., \& Bullmore, E. T. (2011). Cognitive Effort Drives Workspace Configuration of Human Brain Functional Networks. Journal of Neuroscience, 31(22), 8259-8270. https://doi.org/10.1523/JNEUROSCI.0440-11.2011

Kitzbichler, M. G., Smith, M. L., Christensen, S. R., \& Bullmore, E. (2009). Broadband Criticality of Human Brain Network Synchronization. PLOS Computational Biology, 5(3), e1000314. https://doi.org/10.1371/journal.pcbi.1000314

Luck, S. J., \& Vogel, E. K. (2013). Visual working memory capacity: from psychophysics and neurobiology to individual differences. Trends in Cognitive Sciences, 17(8), 391-400. https://doi.org/10.1016/j.tics.2013.06.006

Mance, I. (2015). The Contribution of Alpha Oscillations to Working Memory Processing. Retrieved from https://scholarsbank.uoregon.edu/xmlui/handle/1794/19226

Otto, A. R., \& Daw, N. D. (2018). The opportunity cost of time modulates cognitive effort. Neuropsychologia. https://doi.org/10.1016/j.neuropsychologia.2018.05.006

Paas, F., Tuovinen, J. E., Tabbers, H., \& Gerven, P. W. M. V. (2003). Cognitive Load Measurement as a Means to Advance Cognitive Load Theory. Educational Psychologist, 38(1), 63-71. https://doi.org/10.1207/S15326985EP3801_8

Pelli, D. G. (1997). The VideoToolbox software for visual psychophysics: transforming numbers into movies. Spatial Vision, 10(4), 437-442. https://doi.org/10.1163/156856897X00366 
R. Chialvo, D. (2004). Critical brain networks. Physica A: Statistical Mechanics and Its Applications, 340(4), 756-765. https://doi.org/10.1016/j.physa.2004.05.064

Rouder, J. N., Morey, R. D., Cowan, N., Zwilling, C. E., Morey, C. C., \& Pratte, M. S. (2008). An assessment of fixed-capacity models of visual working memory. Proceedings of the National Academy of Sciences, 105(16), 5975-5979. https://doi.org/10.1073/pnas.0711295105

Sederberg, P. B., Kahana, M. J., Howard, M. W., Donner, E. J., \& Madsen, J. R. (2003). Theta and Gamma Oscillations during Encoding Predict Subsequent Recall. Journal of Neuroscience, 23(34), 10809-10814. https://doi.org/10.1523/JNEUROSCI.23-3410809.2003

Van de Ville, D., Britz, J., \& Michel, C. M. (2010). EEG microstate sequences in healthy humans at rest reveal scale-free dynamics. Proceedings of the National Academy of Sciences, 201007841. https://doi.org/10.1073/pnas.1007841107

Vogel, E. K., \& Machizawa, M. G. (2004). Neural activity predicts individual differences in visual working memory capacity. Nature, 428(6984), 748-751. https://doi.org/10.1038/nature02447

von Wegner, F., Laufs, H., \& Tagliazucchi, E. (2018). Mutual information identifies spurious Hurst phenomena in resting state EEG and fMRI data. Physical Review E, 97(2). https://doi.org/10.1103/PhysRevE.97.022415

Wendt, H., Abry, P., \& Jaffard, S. (2007). Bootstrap for Empirical Multifractal Analysis. IEEE Signal Processing Magazine, 24(4), 38-48. https://doi.org/10.1109/MSP.2007.4286563 
Werner, G. (2010). Fractals in the nervous system: conceptual implications for theoretical neuroscience. Frontiers in Physiology, 1. https://doi.org/10.3389/fphys.2010.00015

Westbrook, A., \& Braver, T. S. (2015). Cognitive effort: A neuroeconomic approach.

Cognitive, Affective, \& Behavioral Neuroscience, 15(2), 395-415.

https://doi.org/10.3758/s13415-015-0334-y 


\section{Supplementary materials}

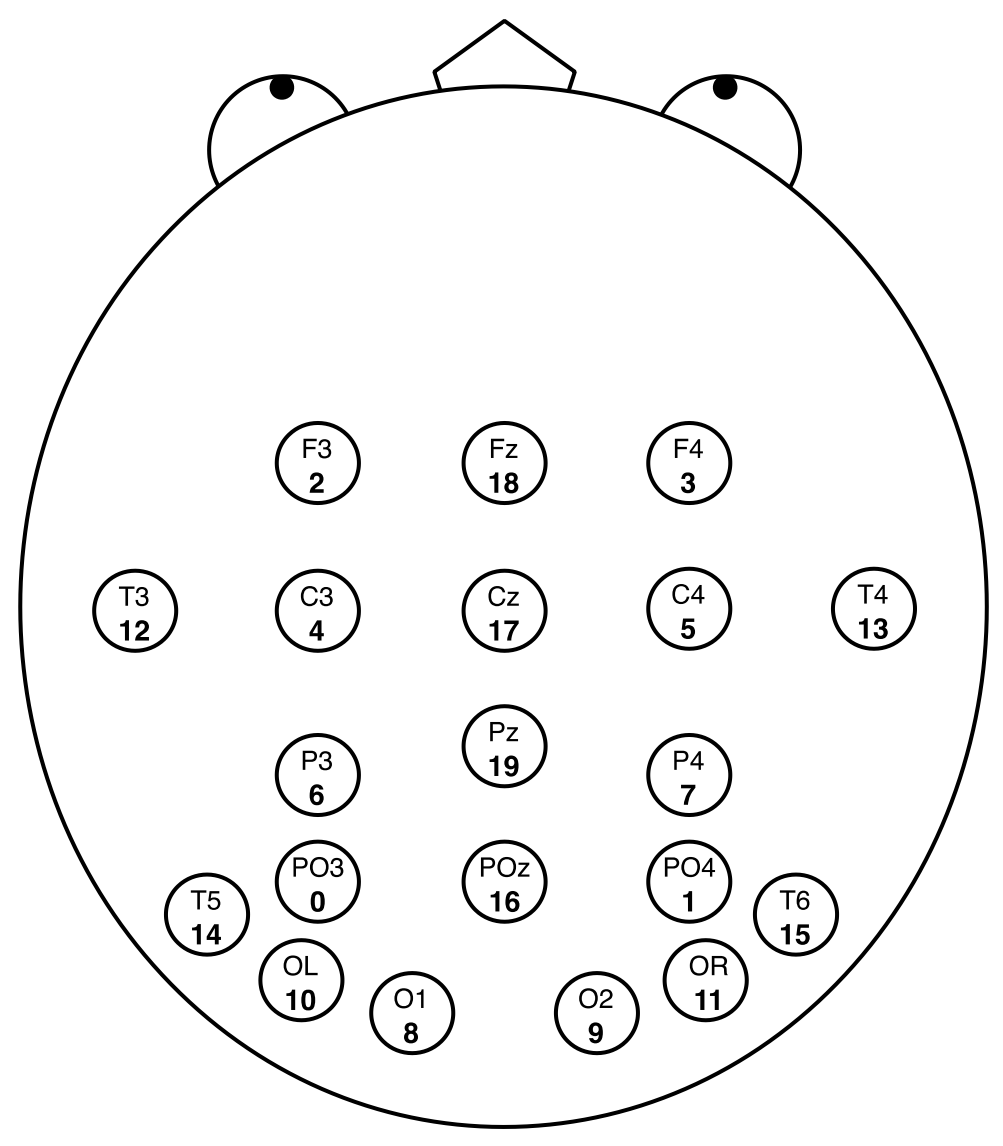

Figure S1. The electrode positions in the experiments. 


\section{Correlations with $\mathrm{H}$ in Experiment 1}

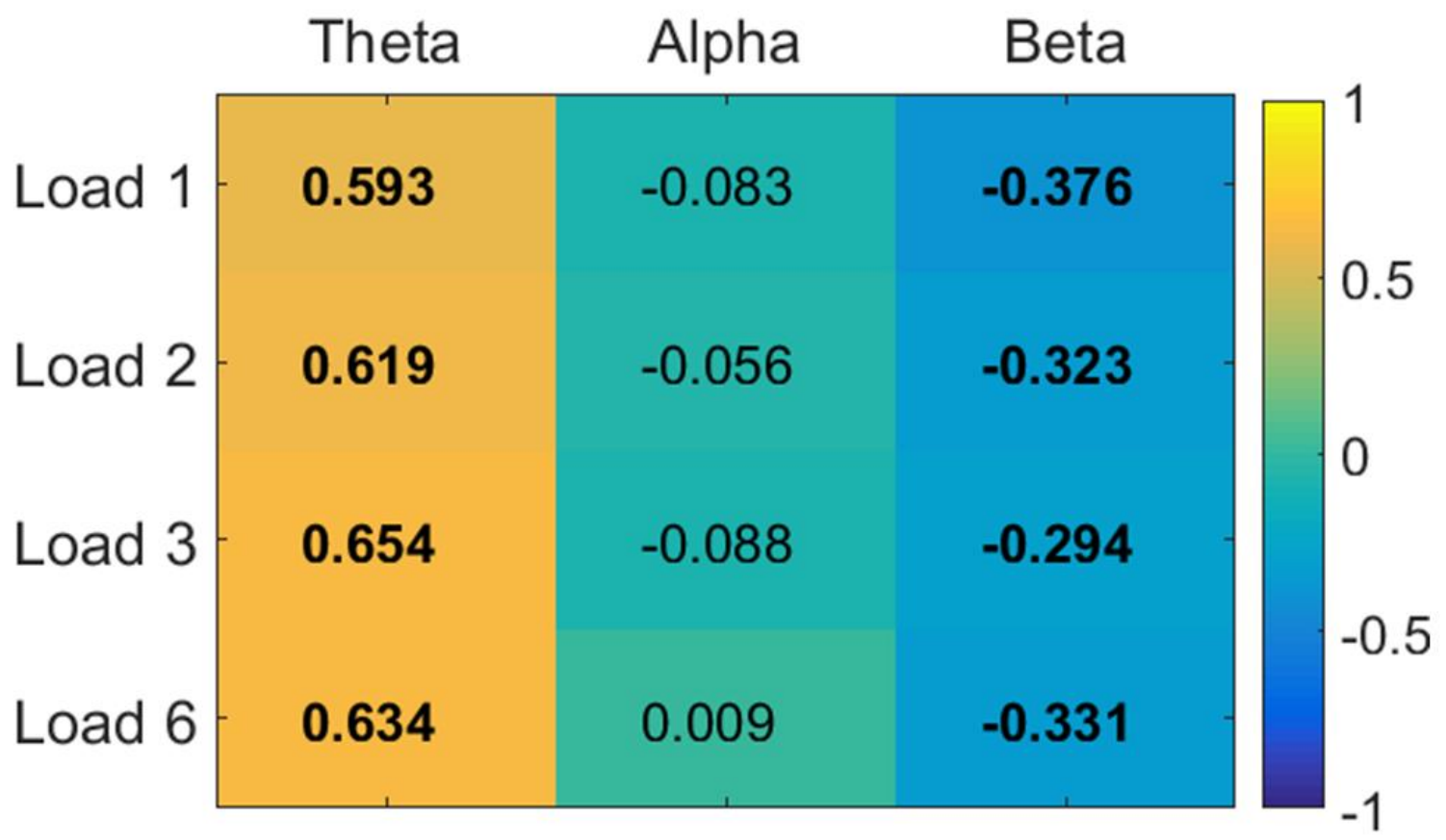

Correlations with $\mathrm{H}$ in Experiment 2

Theta Alpha Beta

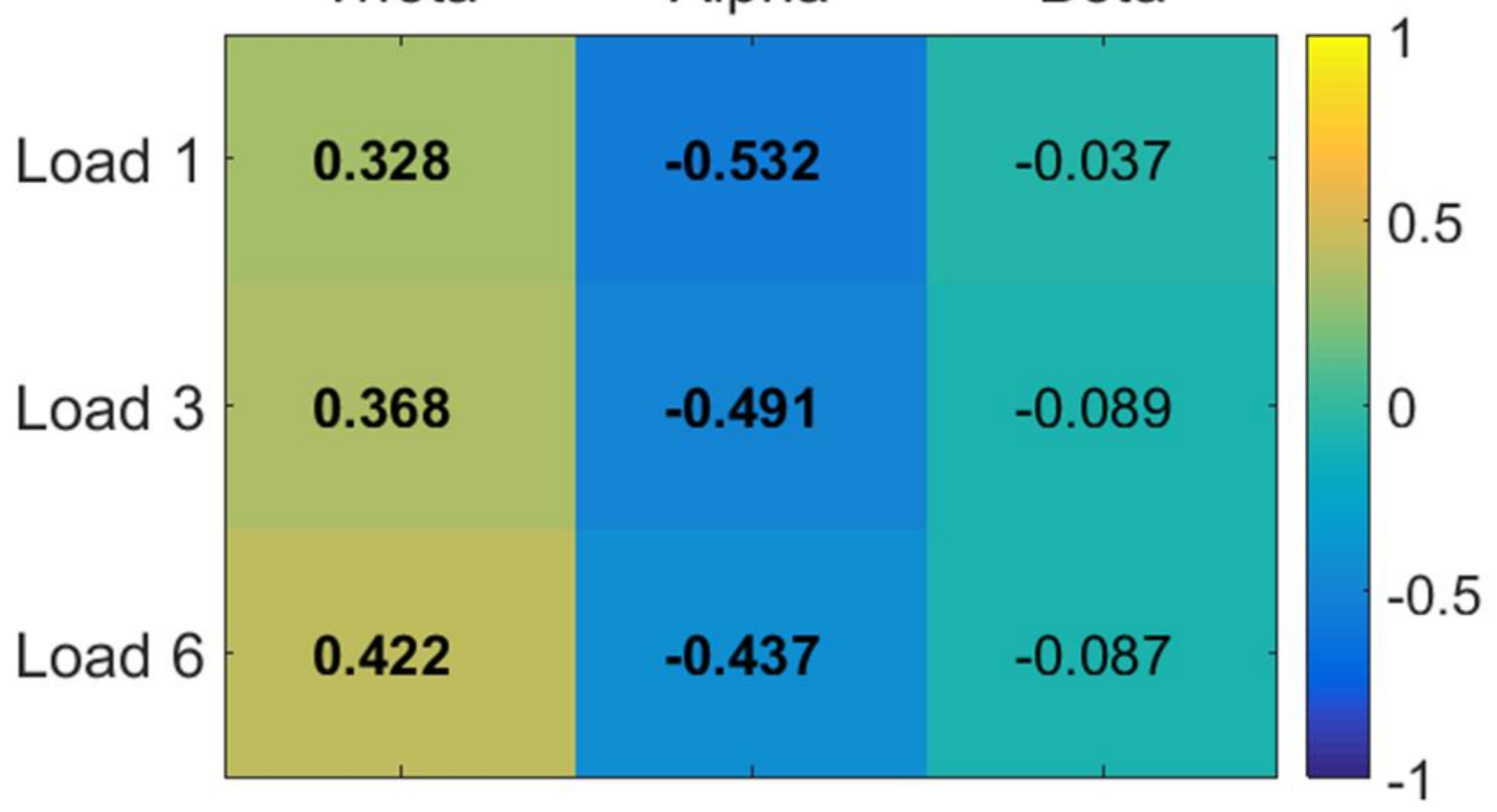

Figure S2. Correlations of theta, alpha, and beta band powers with scale-invariance $(H)$ within each set size in experiments 1 and 2 . 


\section{CCA for theta and beta bands}

Neither theta nor beta band powers had a stable relationship with set size in Experiment 1 (see Figure S3). In experiment 2, only theta band power showed a stable relationship with the set size (Figure S4), but this relationship could not be responsible for the pattern observed for $H$ for two reasons: 1 ) The spatial pattern of the $H$ in in experiment 2 showed a global suppression, whereas there is a more sparse and spatially heterogeneous relationship between theta power and set size (where higher set sizes correspond to less power in central and more power in occipital electrodes), and 2) The strength of the association is smaller for theta compared to $H$ ( $r=0.50$ vs. $r=0.65$, respectively). 

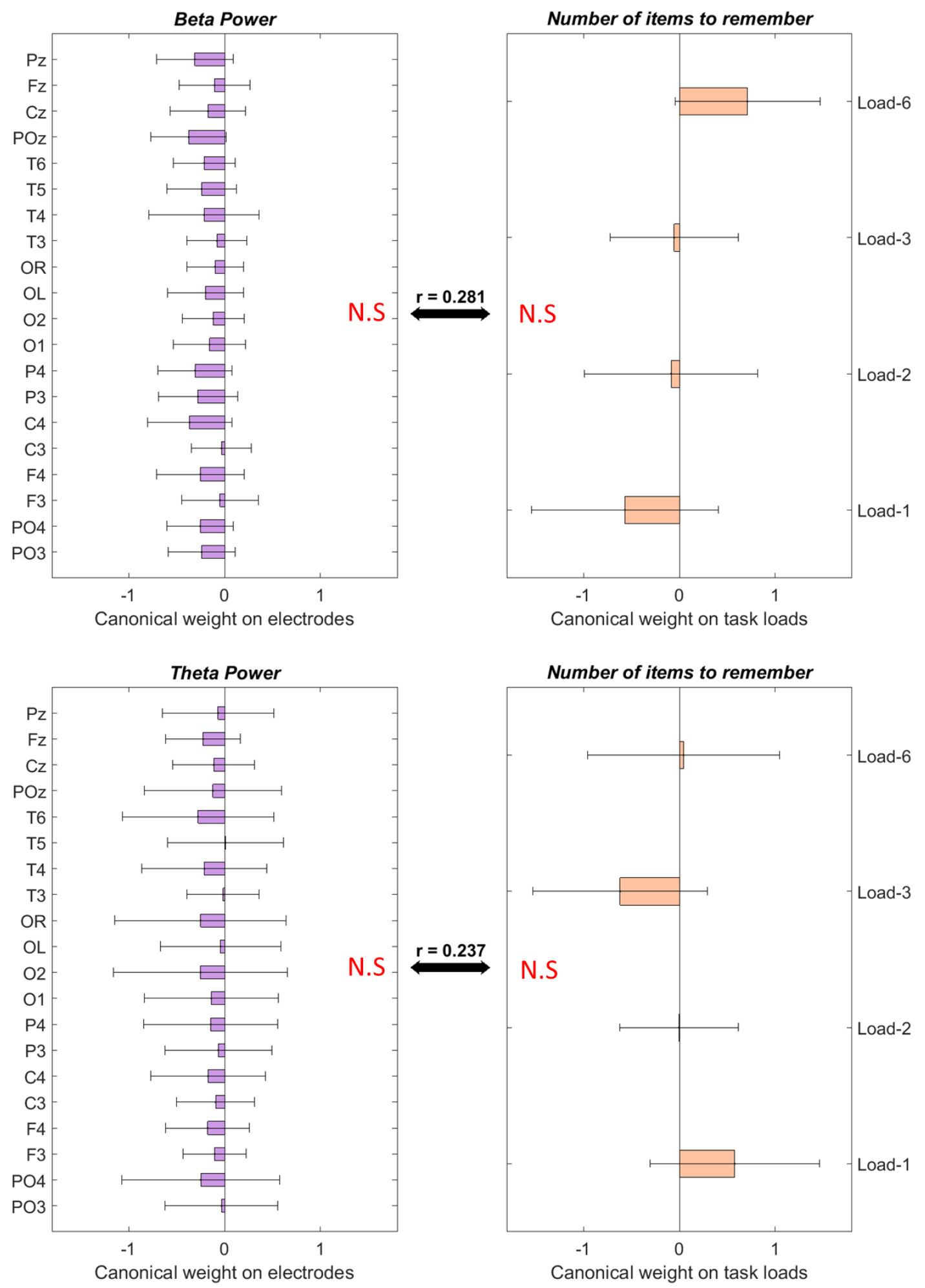

Figure S3. CCA results relating the power of theta and beta band oscillations during retention period to the task load levels in experiment 1. 

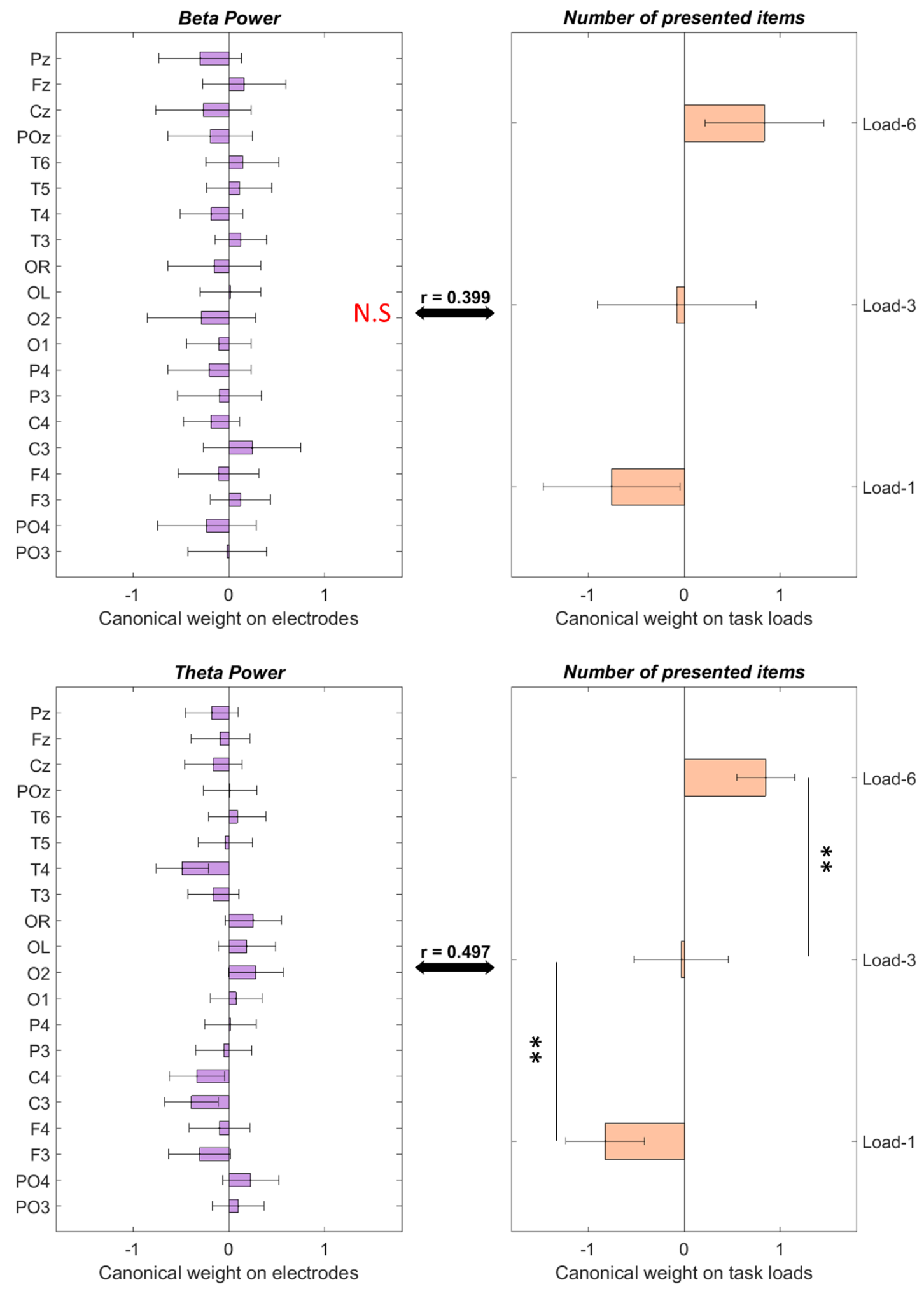

Figure S4. CCA results relating the power of theta and beta band oscillations during retention period to the task load levels in experiment 2 . 